\title{
Trauma Surgery \& Acute Care Open \\ Barriers to and facilitators of a screening procedure for PTSD risk in a level I trauma center
}

\author{
Sacha A McBain, ${ }^{\oplus 1,2}$ Kevin W Sexton, $^{\oplus 3}$ Brooke E Palmer, $^{1}$ Sara J Landes ${ }^{1,2}$
}

- Additional material is published online only. To view please visit the journal online (http://dx.doi.org/10.1136/ tsaco-2019-000345).

'Department of Psychiatry, University of Arkansas for Medical Sciences, Little Rock, Arkansas, USA

2Mental IIIness Research

Education and Clinical Center (MIRECC), Central Arkansas Veterans Healthcare System, Eugene J Towbin Healthcare Center, North Little Rock, Arkansas, USA

${ }^{3}$ Department of Surgery, Division of Acute Care Surgery, University of Arkansas for Medical Sciences, Little Rock, Arkansas, USA

Correspondence to Dr Sacha A McBain, Department of Psychiatry, University of Arkansas for Medical Sciences, Little Rock, AR 72205, USA; smcbain@uams.edu

Received 19 June 2019 Revised 12 July 2019 Accepted 17 July 2019
(C) Author(s) (or their employer(s)) 2019. Re-use permitted under CC BY-NC. No commercial re-use. See rights and permissions. Published by BMJ.

To cite: McBain SA, Sexton KW, Palmer BE, et al. Trauma Surg Acute Care Open 2019:4:e000345.

\section{ABSTRACT}

Background Patients admitted to the hospital after an injury are at a greater risk for developing post-traumatic stress disorder (PTSD) due to the nature of the injury and the traumatic nature of necessary medical interventions. Many level I trauma centers have yet to implement screening protocols for PTSD risk. The goal of the study was to characterize the barriers to and facilitators of implementation of a screening procedure for PTSD risk in a level I trauma center.

Methods We conducted semistructured qualitative interviews with multidisciplinary academic medical center stakeholders $(\mathrm{N}=8)$ including those with clinical, research, teaching, and administrative roles within an urban academic medical center's Department of Surgery, Division of Acute Care Surgery. We analyzed the qualitative data using summative template analysis to abstract data related to participants' opinions about implementation of a screener for PTSD.

Results Participants' general perception of screening for PTSD risk after injury was positive. Identified challenges to implementation included timing of screening, time burden, care coordination, addressing patients with traumatic brain injury or an altered mental status, and ensuring appropriate care after screening. Reported facilitators included existing psychosocial screening tools and protocols that would support inclusion of a PTSD screener, a patient-centered culture that would facilitate buy-in from providers, a guideline-driven culture, and a commitment to continuity of care.

Conclusions This study offers concrete preliminary information on barriers to and facilitators of PTSD screening that can be used to inform planning of implementation efforts within a trauma center. Level of evidence Level $V$, qualitative.

\section{BACKGROUND}

Injury has been linked with comorbid post-traumatic stress disorder (PTSD) and depression. ${ }^{12}$ It is estimated that $2 \%-40 \%$ of patients will report PTSD symptoms up to 12 months after an injury. ${ }^{23}$ PTSD occurrence is one of the strongest correlates of postinjury quality of life $^{4}$ and has been found to negatively impact physical health and increase healthcare utilization. ${ }^{5}$ Patients admitted to the hospital after an injury are at a greater risk for developing PTSD due to the nature of the injury and the acute stress of necessary medical interventions. ${ }^{1}$

Despite the prevalence of PTSD, many level I trauma centers have yet to implement screening protocols. Approximately $7 \%$ of level I trauma centers routinely screen for PTSD compared with $18 \%$ for depression, $43 \%$ for suicidality, $80 \%$ for drug use, and 92\% for alcohol use. ${ }^{6}$ To address this gap in care, the American College of Surgeons Committee on Trauma (ACS-COT) recommends trauma centers screen all trauma patients for PTSD and depression risk. ${ }^{7}$ Abbreviated PTSD screening may be successfully integrated into trauma services and inpatient surgical services of level I trauma centers and has value in predicting and treating those who are likely to develop chronic PTSD. ${ }^{18-10}$ However, there is a paucity of information available on how to implement PTSD screening in level I trauma centers. Given this lack of information, the goal of the current study was to identify and describe the barriers to and facilitators of implementation of a screening procedure for PTSD risk in a level I trauma center.

\section{METHODS}

\section{Setting}

The current study was conducted in an urban academic medical center's Department of Surgery, Division of Acute Care Surgery that holds designations as an American College of Surgeons verified level I trauma center. The trauma center is a 24-bed surgical intensive care unit composed of an interdisciplinary team that provides tertiary medical care to critically ill and injured adult patients throughout the state. In 2018, there were a total of 2598 admissions to the trauma center. Of those 2598 admissions, 809 patients had a Glasgow Coma Scale score greater than 13 and a length of stay longer than 2 days suggesting they may be appropriate for PTSD screening. The table 1 describes the primary injury type and Injury Severity Score (ISS) of those who may be appropriate for screening.

\section{Recruitment}

Multidisciplinary stakeholders $(\mathrm{N}=8)$ were recruited including those with clinical, research, teaching, and administrative roles within the trauma center's inpatient unit and the outpatient follow-up clinic. The sample included three trauma surgeons, two advanced practice nurses, one consultation-liaison psychiatrist, one outpatient nurse, and one member of hospital leadership. Stakeholders were contacted via their medical center e-mail to assess interest in participating in a semistructured interview and were scheduled on an individual basis via e-mail.

\section{Data collection}

Each stakeholder participated in an in-person, individual semistructured qualitative interview to gather initial data regarding the barriers to and 
Table 1 Injury characteristics of patients appropriate for posttraumatic stress disorder (PTSD) screening

\begin{tabular}{lc}
\hline Characteristics & Percent (n) \\
\hline Appropriate for PTSD screening** & \\
\hline No & $68.9(1789)$ \\
\hline Yes & $31.1(809)$ \\
\hline Injury type & \\
\hline Blunt & $84.4(683)$ \\
\hline Penetrating & $13.6(110)$ \\
\hline Burn & $1.7(14)$ \\
\hline Other/unknown & $0.3(2)$ \\
Injury Severity Score (ISS) & \\
\hline Minor (ISS 1-8) & $47.4(384)$ \\
\hline Moderate (ISS 9-15) & $22.5(182)$ \\
\hline Moderate/severe (ISS 16-24) & $10.6(86)$ \\
\hline Severe/critical (>24) & $10.8(87)$ \\
\hline Unknown & $8.7(70)$ \\
\hline
\end{tabular}

* Patients were deemed appropriate for PTSD screening based on their alertness (Glasgow Coma Scale score $>13$ ) and duration of admission (length of stay $>2$ days)

facilitators of implementation of a PTSD screening protocol in their service. The qualitative interview guide was constructed utilizing the Consolidated Framework for Implementation Research (CFIR), ${ }^{11}$ a framework designed to identify constructs and domains that impact implementation of programmes or practices. All interviews were conducted by the principal investigator. The interviews were audiorecorded with permission from participants and transcribed by medical transcription services. This study was provided a non-human subjects research determination by the medical center's institutional review board.

\section{Data analysis}

Summative template analysis, a data abstraction procedure developed by Hamilton, ${ }^{12}$ was used to analyze the data. Two qualitative raters completed the analysis. Prior to beginning, the lead rater developed a prototype summary template with seven broad domains that corresponded with the CFIR-informed interview guide questions: (1) applicability to trauma surgery, (2) fit with existing practices, (3) method for identifying patients for screening, (4) facilitators of implementation, (5) barriers to implementation, (6) training needed by staff, (7) perceived patient and/or family response, and (8) other. See the online supplementary appendix 1 for the CFIR-informed interview guide.

For each interview, both raters independently analyzed the transcript and summarized the content related to participants' recommendations and opinions (eg, if a participant discussed how time was a significant barrier it was summarized in the template as 'time burden'), placing it in the appropriate template category. Especially salient quotes were included in the templates to enrich the data. The raters met to discuss the templates after analyzing each transcript and come to an agreement about what information should be retained in the combined template. The combined template was created by the lead rater and reviewed by the second rater to ensure consensus. Discrepancies in how the content was summarized or categorized were rare and resolved through discussion. This analytic process was repeated for each individual transcript. A rubric was developed to define inductive categories and sub-categories as they emerged to ensure consistency and promote analytic rigor (eg, 'facilitators to implementation' was defined as a domain categorizing any reference to ways to ensure implementation is successful). After analyses were finalized for all transcripts, all the individual summary templates were compiled and summarized into a final study summary template. Raters each reviewed the final study summary template to ensure the validity of the findings.

\section{RESULTS}

Two participants (25\%) reported they were aware of ACS-COT's PTSD screening guidelines prior to being interviewed. Participants' general perception of screening for PTSD risk after injury was positive. All participants believed implementation of a screening protocol would be beneficial and that patients would overall react positively. Participants found PTSD screening aligned with trauma surgery culture, specifically, a commitment to improving the quality of care and a culture of innovation. As one participant stated:

Trauma is a unique group of people...we do things not because we're expecting recognition but because it is right for the patient. I wish we had a statement for our Division. It would be, 'Do the right thing for the patient.'

Primary advantages of implementing PTSD screening identified by participants included improved patient-centered outcomes, increased access to mental healthcare, improved adherence to ACS guidelines, and a greater understanding of prevalence of PTSD in the trauma surgery population. As one participant described it:

Some of these patients are high utilizers of services. They might develop a lot of somatic complaints, and it might be a revolving door for them. If we get them plugged in, we might be able to help them take better care of themselves, not only emotionally but also for physical needs.

The most prevalent barrier to implementing PTSD screening identified by participants was potential system strain, including the possibility of an increased care burden and need for additional referral and follow-up. A participant described system strain in this way:

This would be adding another component and, while it may only take a minute or two, a minute or two when you have 20 or 30 patients to take care of can be overwhelming.

Participants' identified challenges to implementation included timing of screening (eg, at admission or discharge), time burden, care coordination, patients' willingness to engage, and a lack of an established relationship with existing mental health services. The most commonly reported challenges were how to address patients with traumatic brain injury or an altered mental status and ensuring appropriate care after screening (eg, the ability to provide appropriate referrals). One participant elaborated on this difficulty with appropriate referrals by stating that many patients already 'have trouble getting to primary care doctors as it is'. Participants also identified possible solutions to challenges described; see table 2 for more detail.

Reported facilitators of implementation included existing psychosocial screening tools and protocols that would support inclusion of a PTSD screener (eg, electronic medical record trauma navigator tab, tertiary examination), a patient-centered culture that would facilitate buy-in from providers, existing emphasis on guidelines to improve patient care, a commitment to continuity of care (eg, outpatient trauma clinic), staff buy-in, 
Table 2 Challenges and potential solutions for successful implementation of PTSD screening identified by participants

\begin{tabular}{|c|c|}
\hline Challenge & Potential solution \\
\hline $\begin{array}{l}\text { Identifying who to } \\
\text { screen }\end{array}$ & $\begin{array}{l}\text { Use trauma registry } \\
\text { Use a standard approach } \\
\text { Clearly defined protocol for positive screens } \\
\text { Include flexibility for clinical judgement } \\
\text { Make screening unit based } \\
\text { Consider treatment trajectory }\end{array}$ \\
\hline Timing of screening & $\begin{array}{l}\text { Integrate into existing systems (eg, admission navigator, } \\
\text { flow sheet, discharge protocol) } \\
\text { Use existing continuity of care methods (eg, nursing } \\
\text { postdischarge follow-up call) }\end{array}$ \\
\hline Time burden & $\begin{array}{l}\text { Delegate to non-physician staff } \\
\text { Use brief measurements } \\
\text { Integrate into existing systems } \\
\text { Work with database managers to streamline data entry } \\
\text { Develop full-time position to coordinate screening }\end{array}$ \\
\hline Patient engagement & $\begin{array}{l}\text { Staff education } \\
\text { Informational meetings for patients' families } \\
\text { Embed in larger psychosocial assessment }\end{array}$ \\
\hline $\begin{array}{l}\text { Lack of established } \\
\text { relationship with mental } \\
\text { health services }\end{array}$ & $\begin{array}{l}\text { Identify existing mental health services (eg, consultation- } \\
\text { liaison, social work, case management) } \\
\text { Engage existing mental health services in implementation } \\
\text { planning process } \\
\text { Develop a full-time mental health position integrated into } \\
\text { the service }\end{array}$ \\
\hline
\end{tabular}

and leadership support. One participant captured the importance of a leadership support to facilitate change:

\begin{abstract}
You've got to have somebody that's in a high enough position within the organization to say, 'This is a priority; we're doing this' and sort of champion it. I can advocate for this all day long, but without some top cover it's probably not going to go very far. Buy in from the leadership, buy in from whoever is the management over who's going to do this, and the nurses. They have to understand why it's important.
\end{abstract}

Participants also emphasized the importance of a standard approach to screening that minimizes need for clinical judgement and is easily integrated into an existing workflow. A participant described the importance of a standard approach in this way:

From previous experience, we do better with an all or none intervention, meaning that when we set forth a new guideline it's much easier if we say, 'this is our standard approach to every patient.' You can use clinical judgement to deviate but, unless something is happening out of the ordinary, we expect this to be done.

\section{DISCUSSION}

This qualitative study describes trauma surgery stakeholders' attitudes towards implementation of PTSD screening in an urban trauma and acute care surgery service. Previous research has highlighted the importance of screening for PTSD risk after a traumatic injury and demonstrated the feasibility of implementing PTSD screening in a level I trauma center. ${ }^{10}$ The ACS supports efforts to implement screening protocols for PTSD using evidence-based screening tools such as the primary care PTSD screen ${ }^{13}$ and the PTSD checklist. ${ }^{14}{ }^{15}$ Results from the current study suggest positive opinions regarding implementation of PTSD screening among a group of providers interested in doing the preliminary work to move towards implementation of a screening protocol. Implementation of PTSD screening is aligned with values of the trauma surgery culture including an emphasis on patient-centered innovation and an organizational infrastructure based on guidelines. All participants reported they believed PTSD screening would be beneficial to patients and consistent with the culture of their division.

The barriers identified by participants are commonly cited barriers regarding adoption of new clinical guidelines. ${ }^{16}$ There is variable awareness of the PTSD screening guidelines and minimal guidance on how to implement a screening protocol in this setting. Implementation of PTSD screening is needed, and positive opinions and good outcomes of PTSD screening have been demonstrated. ${ }^{17} 18$ The results of the current study suggest a culture that will support implementation of PTSD screening with assistance.

Given the existing barriers and facilitators, a multidisciplinary implementation team including leadership, frontline providers, educators, clinical informatics, and database managers will be needed to successfully implement a screening protocol. Access to a subject matter expert may also be critical for implementation of mental health interventions within a trauma setting. For implementation of any new clinical guideline, the absence of people within the organization that have an intrinsic interest in implementation of the change (ie, 'champions') ${ }^{19}$ can stagnate uptake of new guidelines. This is likely further complicated when non-mental health providers are tasked with implementation of a mental health-related guideline for which they have little content expertise. ${ }^{20}$ As previously mentioned, 92\% of trauma centers are routinely screening for alcohol use compared with 7\% for PTSD. ${ }^{6}$ The success of screening adoption may correlate with the topic's grounding in existing medical training. This was seen anecdotally in the current study. The study trauma center had independently and successfully implemented an alcohol screening protocol, however, had not implemented PTSD screening. Now that interviews have been completed with a subject matter expert, multiple stakeholders are motivated to implement PTSD screening, as indicated by requests for help to implement.

\section{CONCLUSIONS}

This study offers concrete preliminary information on barriers to and facilitators of PTSD screening that can be used to inform planning of implementation efforts within a trauma center. As a qualitative study conducted within a single trauma center, these results are preliminary and may have limited generalizability to other trauma centers. Further research could provide further study of common barriers and facilitators across a range of culturally diverse trauma centers and information on implementation strategies that may have generalizability across centers.

Contributors $S A M C B$ is the corresponding author and contributed to the data collection. SAMCB and SJL contributed to the study concept and design. SAMCB and $B E P$ contributed to the data analysis. SAMCB, BEP, SJL contributed to the writing of the article. KWS and SJL contributed to the critical revision.

Funding The authors have not declared a specific grant for this research from any funding agency in the public, commercial or not-for-profit sectors.

\section{Competing interests None declared.}

Patient consent for publication Not required.

Provenance and peer review Not commissioned; externally peer reviewed.

Open access This is an open access article distributed in accordance with the Creative Commons Attribution Non Commercial (CC BY-NC 4.0) license, which permits others to distribute, remix, adapt, build upon this work non-commercially, and license their derivative works on different terms, provided the original work is properly cited, appropriate credit is given, any changes made indicated, and the use is non-commercial. See: http://creativecommons.org/licenses/by-nc/4.0/. 


\section{REFERENCES}

1. Hunt JC, Sapp M, Walker C, Warren AM, Brasel K, deRoon-Cassini TA. Utility of the injured trauma survivor screen to predict PTSD and depression during hospital admission. J Trauma Acute Care Surg 2017;82:93-101.

2. Zatzick DF, Rivara FP, Nathens AB, Jurkovich GJ, Wang J, Fan M-Y, Russo J, Salkever DS, Mackenzie EJ. A nationwide US study of post-traumatic stress after hospitalization for physical injury. Psychol Med 2007;37:1469-80.

3. Schnyder U, Moergeli H, Klaghofer R, Buddeberg C. Incidence and prediction of posttraumatic stress disorder symptoms in severely injured accident victims. Am J Psychiatry 2001;158:594-9.

4. Zatzick DF, Marmar CR, Weiss DS, Browner WS, Metzler TJ, Golding JM, Stewart A, Schlenger WE, Wells KB. Posttraumatic stress disorder and functioning and quality of life outcomes in a nationally representative sample of male Vietnam veterans. Am J Psychiatry 1997;154:1690-5.

5. Walker EA, Katon W, Russo J, Ciechanowski P, Newman E, Wagner AW. Health care costs associated with posttraumatic stress disorder symptoms in women. Arch Gen Psychiatry 2003:60:369.

6. Love J, Zatzick D. Screening and intervention for comorbid substance disorders, PTSD, depression, and suicide: a trauma center survey. Psychiatric Services 2014;65:918-23.

7. American College of Surgeons. Resources for optimal care of the injured patient. 2014. https://www.facs.org/quality\%20programs/trauma/vrc/resources.

8. Shalev AY, Gevonden M, Ratanatharathorn A, Laska E, van der Mei WF, Qi W, Lowe S, Lai BS, Bryant RA, Delahanty D, et al. Estimating the risk of PTSD in recent trauma survivors: results of the International Consortium to predict PTSD (ICPP). World Psychiatry 2019;18:77-87.

9. Richmond TS, Ruzek J, Ackerson T, Wiebe DJ, Winston F, Kassam-Adams N. Predicting the future development of depression or PTSD after injury. Gen Hosp Psychiatry 2011:33:327-35.
10. Giummarra MJ, Lennox A, Dali G, Costa B, Gabbe BJ. Early psychological interventions for posttraumatic stress, depression and anxiety after traumatic injury: a systematic review and meta-analysis. Clin Psychol Rev 2018;62:11-36.

11. Damschroder LJ, Aron DC, Keith RE, Kirsh SR, Alexander JA, Lowery JC. Fostering implementation of health services research findings into practice: a consolidated framework for advancing implementation science. Implement Sci 2009:4:50.

12. Hamilton AB, Frayne SM, Cordasco KM, Washington DL. Factors related to attrition from Va healthcare use: findings from the National survey of women veterans. J Gen Intern Med 2013;28(S2):510-6.

13. Prins A, Ouimette P, Kimerling R, Camerond RP, Hugelshofer DS, Shaw-Hegwer J, Thrailkill A, Gusman FD, Sheikh Jl. The primary care PTSD screen (PC-PTSD): development and operating characteristics. Primary Care Psychiatry 2004;9:9-14.

14. Weathers FW, Litz BT, Keane TM, Palmieri PA. The PTSD Checklist for DSM-5 (PCL-5) 2019. www.ptsd.va.gov.

15. The American College of Surgeons. Statement on post-traumatic stress disorder in adults. Statements of the college. 2018. https://www.facs.org/about-acs/statements/ 109-adult-ptsd (Cited 3 Jun 2019).

16. Fischer F, Lange K, Klose K, Greiner W, Kraemer A. Barriers and strategies in guideline implementation - a scoping review. Health Care 2016:4:36.

17. Bell N, Sobolev B, Anderson S, Hewko R, Simons RK. Routine versus AD hoc screening for acute stress following injury: who would benefit and what are the opportunities for prevention. J Trauma Manag Outcomes 2014;8:5.

18. Hanley J, deRoon-Cassini T, Brasel K. Efficiency of a four-item posttraumatic stress disorder screen in trauma patients. J Trauma Acute Care Surg 2013;75:722-7.

19. Miech EJ, Rattray NA, Flanagan ME, Damschroder L, Schmid AA, Damush TM. Inside help: an integrative review of champions in healthcare-related implementation. SAGE Open Med 2018;6:205031211877326.

20. Lewis CC, Lyon AR, McBain SA, Landes SJ. Testing and exploring the limits of traditional Notions of fidelity and adaptation in implementation of preventive interventions. J Prim Prev 2019:40:137-41. 